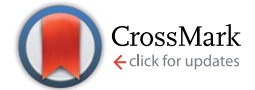

Cite this: RSC Adv., 2016, 6, 60896

Received 1st May 2016

Accepted 11th June 2016

DOI: $10.1039 / c 6 r a 11289 \mathrm{~g}$

www.rsc.org/advances

\title{
Nanomaterial mediated optogenetics: opportunities and challenges
}

\begin{abstract}
Kai Huang, ${ }^{\text {a }}$ Qingqing Dou*b and Xian Jun Loh ${ }^{\star b c d}$
Optogenetics is a promising neuronal modulation strategy in neuroscience, which enables real-time neuromodulation in free-moving animals with high spatiotemporal control. However, it still suffers from several disadvantages, including low penetration of excitation light and the invasiveness of the insertion of the light delivery system. The incorporation of nanomaterials with different properties into optogenetics may bring new opportunities to solve the problems encountered in optogenetics, from the delivery/expression of the optogene to the stimulation/inhibition and follow-up sensing of neural activity. The challenges of nanomaterial-mediated optogenetics are also discussed. This review elaborates on the feasibility of incorporating nanomaterials into optogenetics and analyzes the benefits of nanomaterialmediated optogenetics.
\end{abstract}

\section{Introduction}

Optogenetics, a technique utilizing light to manipulate neural activity and control well-defined events with high specificity and temporal precision, is an emerging and promising neuronal modulation strategy in neuroscience. ${ }^{1}$ It involves genetically engineering nerve cells to produce light-sensitive proteins, and results in the nerve cells either sending or ceasing to send nerve impulses upon exposure to a particular wavelength of light.

${ }^{a}$ Department of Biomedical Engineering, National University of Singapore, Singapore 117576, Singapore

${ }^{b}$ Institute of Materials Research and Engineering, A*STAR (Agency for Science, Technology and Research), 2 Fusionopolis Way, Innovis, \#08-03, Singapore 138634, Singapore. E-mail: lohxj@imre.a-star.edu.sg; douq@imre.a-star.edu.sg; Tel: +6565131612

${ }^{c}$ Department of Materials Science and Engineering, National University of Singapore, Singapore 117574, Singapore

${ }^{d}$ Singapore Eye Research Institute, 20 College Road, Singapore 169856, Singapore
From its first emergence a decade ago, the field of optogenetics has been experiencing high-speed development. Optogenetics has been widely explored in neuroscience research, ${ }^{2-4}$ and has become a promising therapeutic approach for neuronal disorders such as Alzheimer's disease. ${ }^{5}$ Featured as the Method of the year in 2010, Nature Methods introduced optogenetics as "the combination of genetic and optical methods to achieve gain or loss of function of well-defined events in specific cells of living tissue". 6 The typical procedure for optogenetics is as follows, (1) optogene encoded light-gated ion channels are expressed in a specific group of neurons in a live animal or in neuronal cultures before, (2) the activation light is delivered to the light-gated channel to manipulate neuronal activity, and simultaneously (3) electric signal recording or imaging techniques are used to monitor neuronal activity stimulated by light. Combining the advantages of genetic and optical techniques, optogenetics show superior performance over conventional electrical stimulation in neuroscience research,

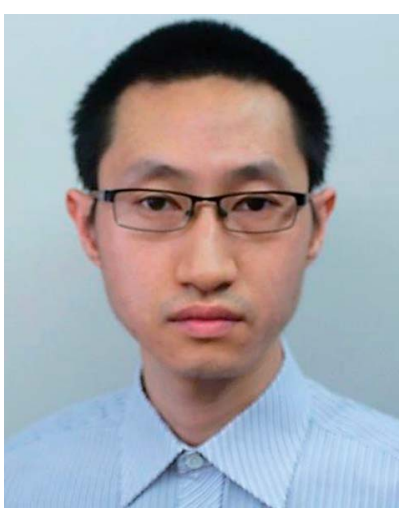

Kai Huang obtained his B.Sc. in materials chemistry (University of Science and Technology of China) and Ph.D. in Biomedical Engineering (National University of Singapore) in 2011 and 2016 respectively. His research focuses on the synthesis and characterization of upconversion nanoparticles and their applications for biomedical applications.

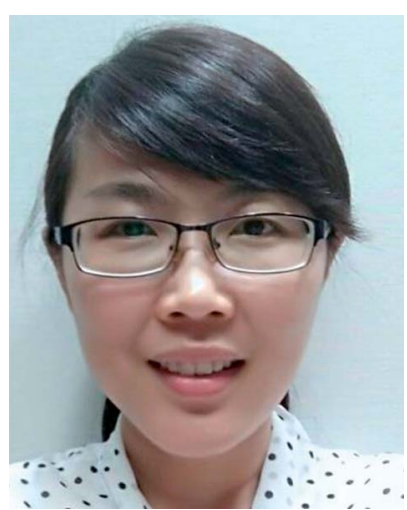

Qingqing Dou received her $P h D$ from National University of Singapore in 2012. After graduation, she joined Institute of Materials Research and Engineering, $A^{*} S T A R$ Singapore. Her research interest lies in upconversion fluorescent nanoparticles, functional polymer hydrogels and exploring their biomedical applications. 
especially for real-time neuromodulation in freely moving animals, by providing high spatiotemporally control of neuronal activities with minimum invasion. ${ }^{7}$

Important progress had been achieved in optogenetics in the past decade. These achievements were mainly focused on optimization of four components in optogenetics, namely the light-gated ion channel, light delivery system, sensor of neural activity, and optogene delivery system. Genetically modifying the light-gated ion channel enables it to be stimulated with longer wavelength light (up to $590 \mathrm{~nm})^{8,9}$ so that manipulation is possible in deeper tissue. Non-contact manipulation of deeper tissue also minimize invasion originated from the light delivery device. Another milestone in engineering of light-gated channel was the discovery of light sensitive inhibitor. This discovery, combining with light sensitive stimulator, enables the modulation switch for neural activities in both stimulation and inhibition manner. ${ }^{\mathbf{1 0}, 11}$ The two-way switch confirms the causality of both "sufficiency" (stimulation) and "necessity" (inhibition) between neural circuits and animal behaviour. Development of the light delivery system focused mainly on the improvement of light sources or the way of light delivery. Due to the inherent light scattering property of the mammalian skull, the penetration of external light is limited, rendering it unable to reach the neuron for stimulation. There has thus been a need for the development of the light source, which has been evolving from a head-mounted LED $^{12,13}$ to a thin optical fiber, ${ }^{14}$ further to a wireless implanted small LED device. ${ }^{15,16}$ Such advances have allowed for improved optogenetics in terms of minimized invasion and increased precision in both temporal and spatial control. Besides, wavefront shaping technique was also developed for spatiotemporal regulation of cells expressing fibroblast growth factor receptor 1 (optoFGFR1) lying beyond the skull by controlling the wavefront of an excitation beam. ${ }^{17}$ Efforts have been made on development of optical neural activity sensors to achieve higher spatiotemporal resolutions

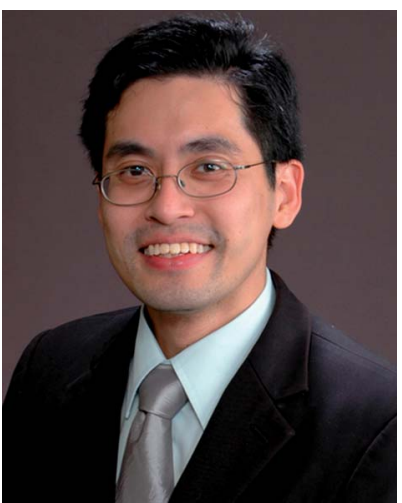

Dr Xian Jun Loh is a polymer chemist working in the interdisciplinary field of biomaterials. He is currently the Programme Manager of the $A^{*} S T A R$ Personal Care Programme. He is concurrently the Programme Manager of the Consumer Care Programme at the Institute of Materials Research and Engineering (IMRE) and an Assistant Professor at the National University of Singapore (NUS). $\mathrm{He}$ is also an adjunct scientist at the Singapore Eye Research Institute. His main research interests are in the design of supramolecular and stimuli-responsive polymers and hydrogels for biomedical and personal care applications. Currently, he is the author and co-author of 112 journal papers, 14 patents, 20 book chapters and 4 books, publishing mainly in the area of biomaterials. over the traditional electrophysiology mapping. Among these optical sensors, ion-sensitive (such as to $\mathrm{Ca}^{2+}$ and $\mathrm{Cl}^{-}$) indicators $^{18}$ and voltage-sensitive phosphatase ${ }^{\mathbf{1 9 , 2 0}}$ attracted the most interest, due to their capability of monitoring the direct changes of neuronal activity. For optogene delivery, genes that contain light-activated mechanism for membrane proteins (such as opsin family genes) are encoded in specific sets of neurons, which results in the expression of the light active membrane protein by the neurons. As opsin family genes are usually extracted from microbes, the delivery and the precise expression of these genes in the target neurons are difficult. ${ }^{21}$ It is essential to explore method for precise optogene delivery and expression with minimized side effects. ${ }^{22,23}$

Despite the significant achievements, several key issues are still remaining in optogenetics. Two out of above-mentioned four components in optogenetics correspond to the light source, i.e. light-gated ion channel and light delivery system. Currently, light source used for optogenetics faces two main problems: the unsatisfactory penetration depth, and the invasive procedure to insert the light source. Light-gated channels are mostly reactive to visible light, but penetration of visible light into tissue is limited. It is favourable to shift excitation wavelength to the range of tissue optical window (from 650 to $1350 \mathrm{~nm}$ ) for deeper penetration. The third problem lies in neural activity sensors. The current ion/voltage sensitive optical sensors are suffering from problems such as slow response, low signal/noise ratio, or dim fluorescence. ${ }^{\mathbf{1 9 2 4 , 2 5}}$ The optogene delivery and expression is also lack of specificity to cell-type, due to the transgene might leak to surrounding light-sensitive neurons. The leakage and subsequent unspecific expression largely decreased the specificity of optogene transfection. ${ }^{22,23,26}$ Cross-discipline endeavours from neuroscience, materials engineering and bioengineering are required to solve the above problems.

\section{Opportunities of nanomaterials in optogenetics}

Recent advance in nanotechnology enables the controllable synthesis of nanomaterials with desirable optical, electrical, thermal properties and proper bio-functionalization. Combining the unique advantages of various nanomaterials with elaborate designs, these nanomaterials can be utilized in optogenetics, from optogene delivery to the stimulation/ inhibition of neural activities and the follow-up neural activities sensing process (Fig. 1).

\subsection{Nanomaterial-mediated stimulation and inhibition}

The most important improvement brought about by nanomaterials is the revolutionary way of stimulating/inhibiting the light-gated channel. As a powerful toolkit for gain and loss of function test in neuroscience research, stimulation/inhibition of neural activities received the greatest research interest in the development of optogenetics. In order to provide a better stimulation strategy, admittedly, notable progress has been achieved as stated above. However, currently the sole 


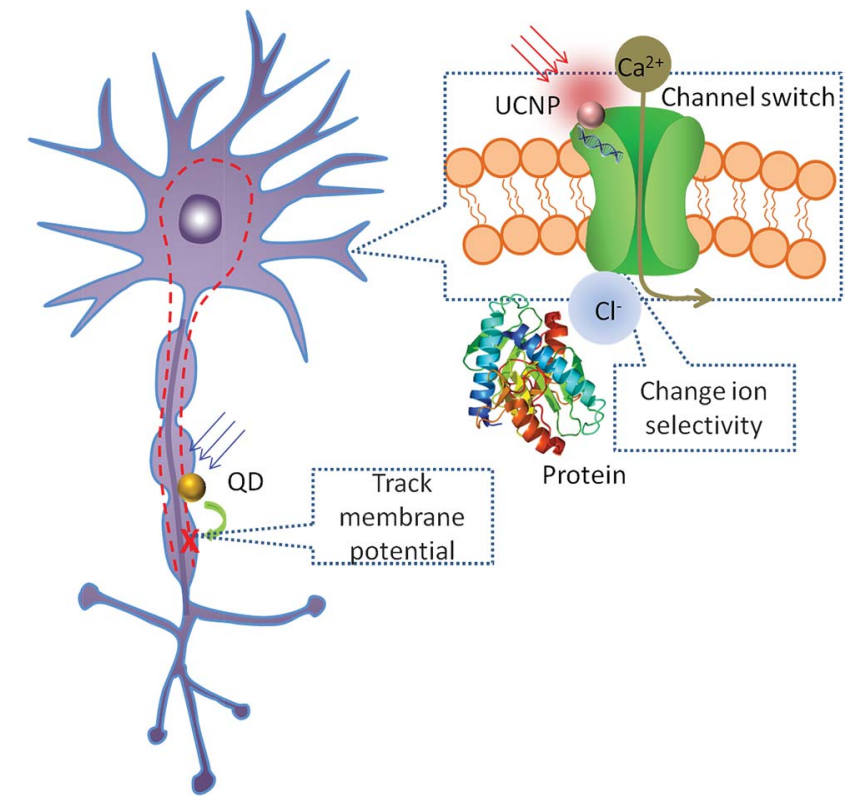

Fig. 1 Schematic of nanomaterials mediated optogenetics.

optogenetics technique is still suffering from the shallow penetration of the excitation light, which is constrained within the visible wavelength range,,$^{9,27,28}$ and its consequent problem of the invasiveness of the light source. This situation can be improved by employing nanomaterials that responding to stimulations that penetrate deeper tissue (such as NIR, ultrasound, X-ray and magnetic field), where nanomaterials serve as nanotransducer for optogenetics. The nanotransducer absorbs excitation energy and emits blue or green light to stimulate the light-gated channels, thus greatly enhancing the workable depth of optogenetics. ${ }^{29-32}$ By acquiring a deeper penetrated excitation source, invasiveness of the procedure can be largely minimized. Moreover, the stimulation is not limited to light, considering the recent discovery of the thermal-sensitive ion channels. ${ }^{33-35}$ The flexibility of surface functionalization of a nanotransducer enables specific binding to the neurons of interest, thus the stimulation/inhibition will be precisely located to the target neurons. As a result, combined with the cell-type selective genetic technology, a better spatial resolution will be achieved.

\subsection{Neuronal activity nanosensors}

Nanomaterials can also serve as the nanosensors to read out the neuronal activity in optogenetics. In optogenetics, electrophysiology test is first conducted to confirm the successful optical manipulation of the localized neuronal activity. ${ }^{\mathbf{1 , 5}, 36}$ Once confirmed, gain and loss of function test can be done through this optical manipulation. At this stage, several methods will be applied to read out the possible corresponding outputs to confirm the causality and further confirm the hypothetical neural circuit. Currently, specific animal behaviour, such as body wall muscle contraction of $C$. elegans, is usually used as the readout at the behaviour level. ${ }^{37,38}$ However, since animal behaviour is a macroscopic response and requires the coordination functioning of complex neuron networks, behaviour test is considered an indirect proof in neuroscience. ${ }^{39}$ At the cellular level, calcium imaging and electrophysiology have good spatial and temporal resolution respectively, and are the two major readout methods in neuroscience. ${ }^{\mathbf{1 8 , 1 9 , 2 4 , 3 6 , 4 0}}$ However, since the response time for the calcium indicator is usually longer than the interval between two optogenetics stimulations, it is not a feasible indicator to read out a rapid stimulation test at a high frequency. ${ }^{18,24,41,42}$ Moreover, blue light or two-photon infrared light is normally used as the excitation light for calcium indicator, which may also excite the channel membrane proteins, thus causing interference to the stimulation. ${ }^{\mathbf{4 3}, 44}$ Electrophysiology is the most popular microscopic readout method available for optogenetics. However, although it is sufficient to sense localized neurons, the spatial resolution of electrophysiology is largely limited by the number of detectors applied to the neural circuit or a larger brain domain. ${ }^{45}$ If luminescent nanomaterials were introduced to the experiments, the spatiotemporal resolution for mapping of the neuronal activities will be enhanced. Currently, several types of voltage-sensitive (such as voltage sensitive quantum dots, ${ }^{46-48}$ plasmonic nanostructures ${ }^{\mathbf{4 9 , 5 0}}$ and polymers $^{51}$ ) or ion-sensitive (such as ion-sensitive metal-semiconductor $^{52,53}$ and gold nanoparticles ${ }^{54}$ ) luminescent nanomaterials have been developed as imaging agents, which can be applied to the mapping of neuronal activities.

\subsection{Nanocarrier for optogene delivery}

Nanomaterials have been widely used in common gene delivery, ${ }^{55-63}$ which may also be modified for optogene delivery. As for the choice of a gene delivery vector, the carriers with the highest gene transfection efficiencies, viruses, suffer from inherent drawbacks, such as immunological problems and insertional mutagenesis. ${ }^{64}$ Nanomaterials have their own unique advantages for gene delivery such as facile functionalization, chemical and thermal stability, and have been reviewed thoroughly elsewhere.58,65 For example, upconversion nanoparticles surface modified with mesoporous silica showed better gene delivery efficiency compared to conventional methods as a result of the photochemical internalization induced by the delivery system. This photochemical internalization helps to disrupt the phagocytosis, and thus protecting the delivered gene from being cleaned out of the target cell. ${ }^{66}$ The system also enables photo-controlled gene expression, contributing to a more precise localized expression of the lightgated ion channel, which will be of great help for the targeted manipulation of neural activity.

\section{Nanomaterials applied in optogenetics}

Nanotechnology could provide approaches to develop precision tools for optogenetics by chemical control, such as modification with specific functional groups to target intended locations and so on. The remote control of neuronal activities in optogenetics is realized by manipulating light-activated ion channels. Light 
application evokes neuronal firing in neurons that express lightactivated ion channels. Therefore, nanomaterials which are optical sensitive or exaggerating electrical signals are possible candidates for optogenetic applications. Optical sensitive nanoparticles can be used to artificially stimulate neurons. Electrical sensitive nanoparticles can be used to detect the optogenetic responses. Current studies on optogenetics with nanomaterials are discussed further below.

\subsection{Upconversion nanoparticles}

Upconversion nanoparticles (UCNPs), which absorb NIR light and emit UV-visible light, have gained numerous interests among biomedical researchers due to NIR light exhibiting less scattering and deeper penetration depth into tissue. In optogenetics, neurons are controlled by the exogenous expression of light-sensitive ion channels embedded in the neuronal plasma membrane,${ }^{67}$ which are usually responsive in UV-visible range. By employing UCNPs as light transducer to activate light-gated ion channels, the optogenetic operation window can be shifted to NIR wavelength with deeper penetration, addressing the problems of using visible light for excitation. ${ }^{68-72}$ However, it should be noted that conventional UCNPs exhibit a narrow excitation band around 975-980 $\mathrm{nm}$, thus suffering from strong water absorption. In addition, the blue emission involves three or four photons absorption in upconversion process, which is especially weak. These two factors constrained the application of UCNPs in optogenetics. ${ }^{73,74}$ A group of effort have been made to enhance the blue emission of UCNP..$^{72,75-78}$ For example, Han et al. enhanced the blue emission of UCNPs through intensive sensitizer ions $\left(\mathrm{Yb}^{3+}\right)$ doping, which was favorable for multiphoton emissions; the nanoparticle was further protected with a layer of $\mathrm{CaF}_{2}$ coating to resist surface quenching in aqueous medium. ${ }^{78}$ Zhang et al. enhanced blue emissions of UCNP by employing pulse modulation as excitation source to achieve efficient optogenetic neuromodulations. ${ }^{72}$ In order to shift the excitation band towards biological window where showing less absorption by tissue, Han et al. designed UCNP with $\mathrm{Nd}^{3+}$ as the sensitizer, which exhibiting excitation band of
$800 \mathrm{~nm}$ suitable for deeper penetration in biological samples. ${ }^{79}$ Then upconversion efficiency of Nd-sensitized UCNPs has been further enhanced through fabricating into core-shell structures. ${ }^{80-83}$ On the other hand, NIR dyes were also incorporated as sensitizers to tune UCNPs with more flexible excitation band (from $780 \mathrm{~nm}$ to $850 \mathrm{~nm}$ ). ${ }^{\mathbf{8 4 , 8 5}}$ The advances in nanomaterial enables UCNPs with preferable properties for optogenetics applications.

In 2011, Deisseroth et al. ${ }^{86}$ filed a patent application on applying UCNPs in optogenetic. In 2013, Han et al. proposed to develop a wireless optogenetic technique with UCNPs in a grant proposal. ${ }^{87}$ Successful UCNP-mediated optogenetic implementation was demonstrated by Lee's group ${ }^{88}$ and Han's group ${ }^{32}$ independently. Lee et al. reported $980 \mathrm{~nm}$ NIR-mediated optogenetic control of neural activity employing hybrid UCNPS to stimulate neurons infected with channelrhodopsin-2 (ChR2), ${ }^{88}$ which is responsive to $470 \mathrm{~nm}$ light. As shown in Fig. 2, $\mathrm{NaYF}_{4}: \mathrm{Yb}^{3+}, \mathrm{Tm}^{3+}$ UCNPs embedded poly(lactic-co-glycolic acid) (PLGA) $0.5 \mu \mathrm{m}$ films were used as underlying culture scaffolds for neurons. The UCNPs converted NIR light into blue light as internal excitation light sources, subsequently activated ChR2-expressed neurons. The neurons generated time-locked, sustained naturalistic impulses with millisecond resolution in response to $980 \mathrm{~nm}$ light pulses at $1 \mathrm{~Hz}, 5 \mathrm{~Hz}$ and $10 \mathrm{~Hz}$.

Han et al. demonstrated the first in vivo remote control of $\mathrm{Ca}^{2+}$ dependent gene expression and photo-modulate immune response with a UCNPs based optogenetic platform (termed "Opto-CRAC", Fig. 3). ${ }^{32}$ Immune cells were firstly genetically engineered to enable $\mathrm{Ca}^{2+}$ gate-controlling protein sensitive to blue light. Blue emission of $\mathrm{NaYF}_{4}: \mathrm{Yb}, \mathrm{Tm} @ \mathrm{NaYF}_{4}$ UCNPs upon $980 \mathrm{~nm}$ was used to on/off of $\mathrm{Ca}^{2+}$ gates, and further induced an immune response. The photosensitive module, LOVSoc, can reversibly generate both sustained and oscillatory $\mathrm{Ca}^{2+}$ signals through the adjustment of pulse and intensity of light input. OptoCRAC functionalized as a genetically-encoded "photoactivatable adjuvant" in a mouse model of melanoma. Recently, another work by Han et al. used $800 \mathrm{~nm}$ NIR light to activate Channelrhodopsin in hippocampal neurons, which were cultured on thin films of poly(methylmethacrylate) embedded with dye-sensitized core/

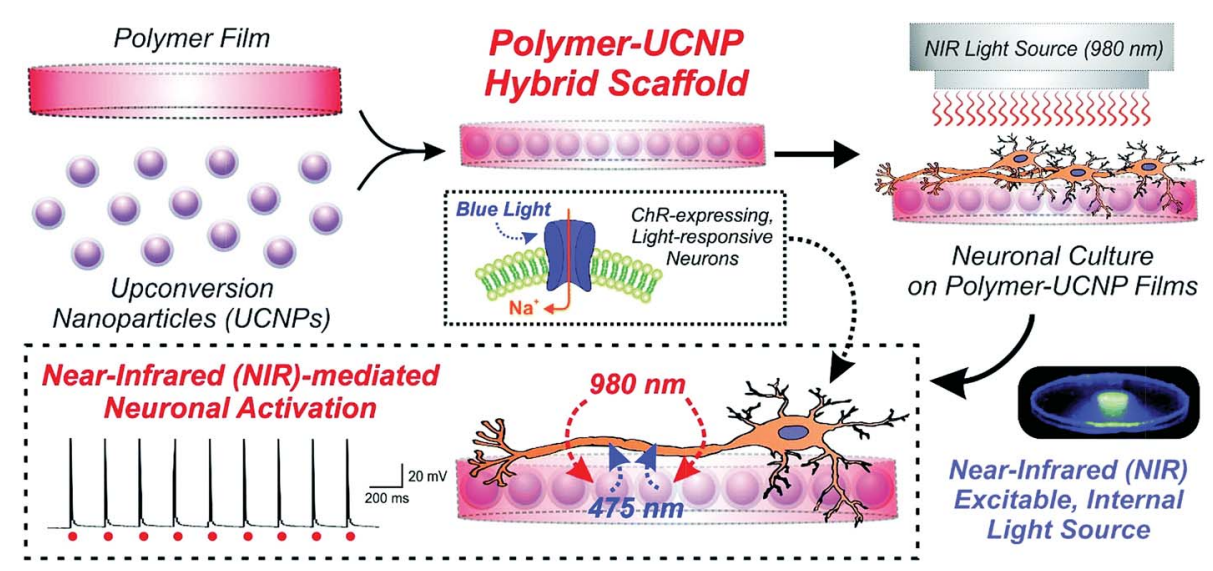

Fig. 2 Schematic diagram depicting the generation and application of polymer-UCNP hybrid scaffolds for optogenetic neuronal activation. Reprint from ref. 88 with permission from The Royal Society of Chemistry. 

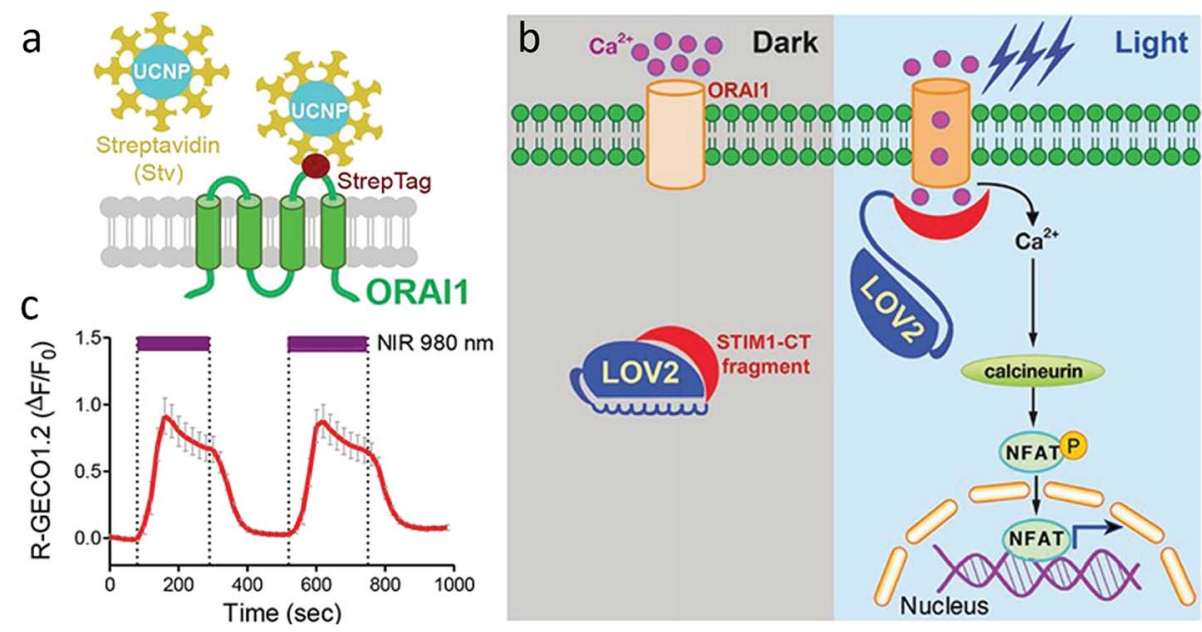

Fig. 3 (a) Schematic showing the interaction of streptavidin-conjugated UCNPs to engineered ORAI1 channels in the plasma membrane of HeLa cells. (b) Schematic of light-operated $\mathrm{Ca}^{2+}$ entry though engineered Opto-CRAC channels. (c) NIR light-induced reversible $\mathrm{Ca}^{2+}$ influx reported by red $\mathrm{Ca}^{2+}$ sensor, R-GECO1.2 in HeLa cells. $980 \mathrm{~nm}$ laser power density: $30 \mathrm{~mW} \mathrm{~mm}{ }^{-2}$. Reprint with permission from ref. 32.

active shell UCNP. ${ }^{89}$ Flexibility in selecting excitation light sources was achieved by using dye to sensitize UCNPs to acquire deeper tissue penetration and minimize heating problem.

Several other groups are also contributing to this field. Yawo et al. demonstrated optogenetic manipulation of neural activities that used $\mathrm{NaYF}_{4}: \mathrm{Sc}^{3+}, \mathrm{Yb}^{3+}, \mathrm{Er}^{3+} @ \mathrm{NaYF}_{4}$ UCNP to activate mVChR1 (ref. 29) and its variants green-light absorbing C1V1 (ref. 29 and 90) with $976 \mathrm{~nm}$ NIR light. UCNP transferred NIR light to visible range that can stimulate C1V1 or mVChR1 to generate a photocurrent in the cells (Fig. 4). The resting potential of the cell membrane is usually $-40 \mathrm{mV}$ to $-70 \mathrm{mV}$, a depolarization of $5-10 \mathrm{mV}$ is required to evoke an action potential. They also observed that NIR irradiation depolarized the membrane potential of the $\mathrm{C} 1 \mathrm{~V} 1 / \mathrm{mVChR} 1$-expressing neurons to evoke surplus action potentials due to relatively slow off kinetics of C1V1 or mVChR1.

Zhang et al. utilized advanced quasi-continuous wave excitation strategy to enhance the luminescence intensity of UCNP nanotransducers without obvious heating effects for efficient optogenetic neuromodulations. ${ }^{72}$ They achieved the first NIR- triggered touch-akin reversal behavior through UCNPmediated optogenetic manipulations in C. elegans model expressing ChR2 in its mechanosensory neurons (Fig. 5).

UCNPs emission tuning and enhancement have been carefully studied in the past decade, strategies such as varying of dopants in the crystalline ${ }^{77,80,91}$ and core-shell structure. ${ }^{\mathbf{8 1 , 8 2 , 9 2}}$ Ease of design UCNPs enables stimulation of wide range of photoresponsive ion channel channelrhodopsins for excitatory or inhibitory by carefully selecting preferable UCNPs. ${ }^{39}$ It is also possible to realize multiplex stimulation.

\subsection{Quantum dots (QD)}

Another big category of optical nanomaterials-quantum dots (QD), has also been applied in optogenetics. As early as in 2002, Maxime et al. used QDs to track individual glycine receptors (GlyRs) and analyze their lateral dynamics in the neuronal membrane of living cells from milliseconds to minutes. ${ }^{93}$ Recently, it was discovered that photoluminescence (PL) of certain QDs was suppressed upon applying an electric field.
A

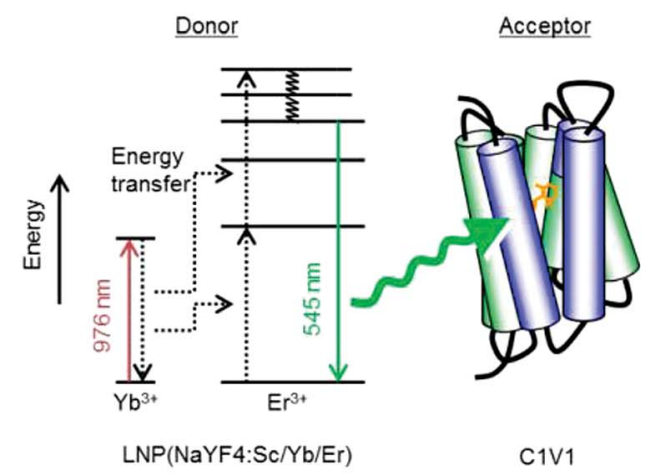

B

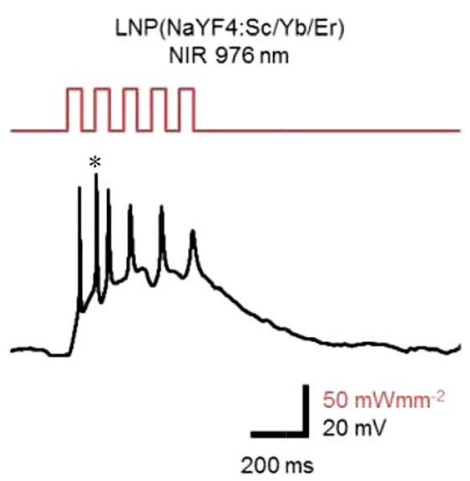

Fig. $4 \mathrm{NaYF}_{4}: \mathrm{Sc}, \mathrm{Yb}$, Er for NIR optogenetics. (A) The visible light emitted from donor $\mathrm{NaYF}_{4}: \mathrm{Sc}, \mathrm{Yb}, \mathrm{Er}$ through upconversion of NIR light is absorbed by the acceptor (C1V1) to open the channel. (B) NIR flash-dependent firing of a cortical neuron through NIR optogenetics system. * denotes the asynchronous action potential. Reprint with permission from ref. 90. 

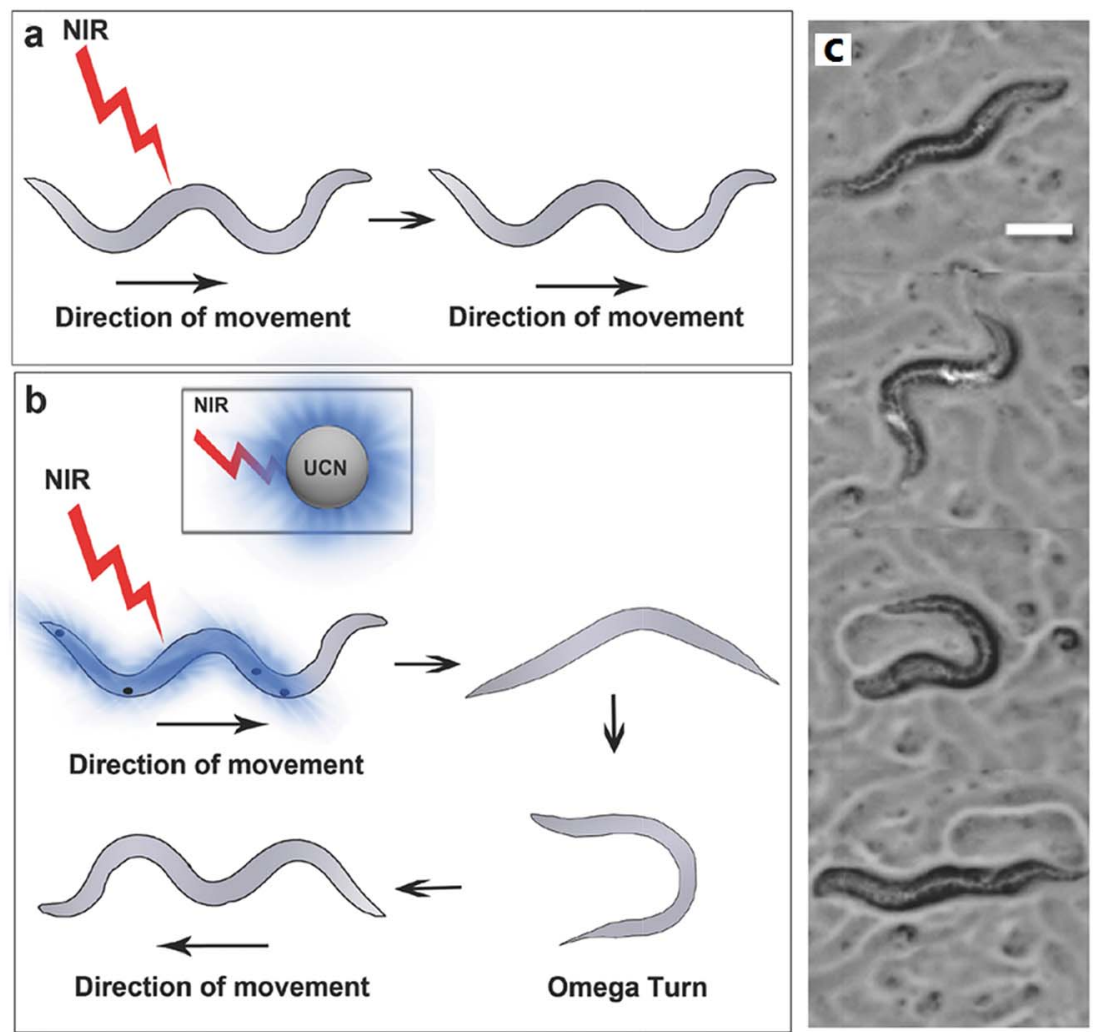

Fig. 5 Quasi-continuous wave near-infrared excitation of upconversion nanoparticles for optogenetic manipulation of $C$. elegans. Schematic showing differences in movement behavior in worms expressing ChR2 in their mechanosensory neurons (a) when exposed to NIR light alone (no UCNPs) and (b) when exposed to NIR light in the presence of UCNPs. (c) Representative images (video stills) showing the reversal process in the UCNP + NIR group. Reprint with permission from ref. 72.

Delehanty demonstrated for the first time that PL suppression of QD could be used in real-time tracking action potential profile of electrically excitable cells like firing neuron (Fig. 6). ${ }^{46}$ Ensemble of CdSe/ZnS QDs or CdS/ZnSe QDs presenting in applied external electric field were used to emulate QDs loaded in a cell's plasma membrane. The number of charged QDs was proportional to the PL quenching. CdS/ZnSe QDs were found to be more sensitive than CdS/ZnS QDs. Theoretically membrane potential could be precisely tracked by $\mathrm{CdS} / \mathrm{ZnSe}$ QDs in a millisecond time scale with an observed $\sim 5 \%$ PL change over a -55 to $+20 \mathrm{mV}$ range. Similarly, other materials showing

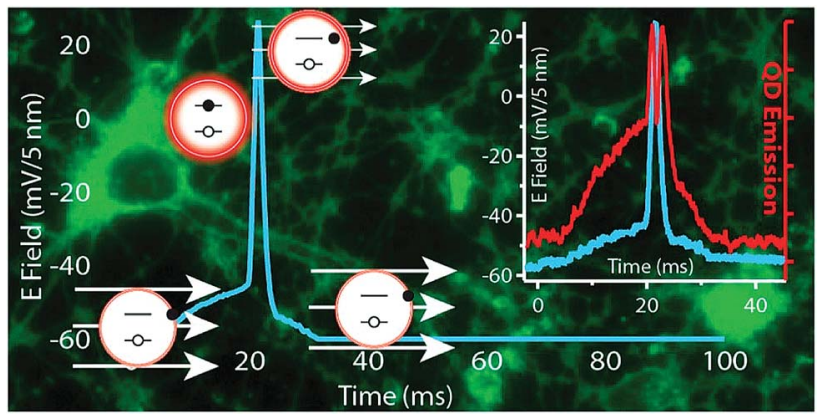

Fig. 6 Quantum dots as voltage-sensitive cellular imaging probes for the readout of neuron activities. Reprint with permission from ref. 46. electrical sensitive photoluminescence can also be used in readout procedure.

Other than serving as the voltage-sensitive imaging probes, the change of electric dipole moments of quantum dots upon excitation could be detected by the closely located voltagegated ion channel, inducing neuron stimulation without genetically engineered light-gated channels. Lin et al. ${ }^{94}$ demonstrated of activation $\mathrm{K}^{+}$and $\mathrm{Na}^{+}$channels and photostimulation of cortical neurons cultured on CdTe or CdSe QDs film (Fig. 7a). Excitation of the QDs films by $550 \mathrm{~nm}$ wavelength light could cause the cell membrane to depolarize, evoking multiple action potentials (Fig. 7b). ${ }^{94}$ Membrane potential and ionic currents were detected when illuminating with $430 \mathrm{~nm}$ light. It should be noted the strength of the sensitized electric field at the cell membrane is prominently dependent on the proximity of the QDs to the membrane. On the other hand, the perturbation of QD also depends on identity of ion channels near the QDs. The above two factors would limit the efficiency of photostimulation. Materials exhibiting large photo-induced electric dipole moments are demanded for this application.

\subsection{Other nanomaterials}

Other than targeting light-gate channels, nanomaterials also demonstrated their ability to active other types of ion channels 

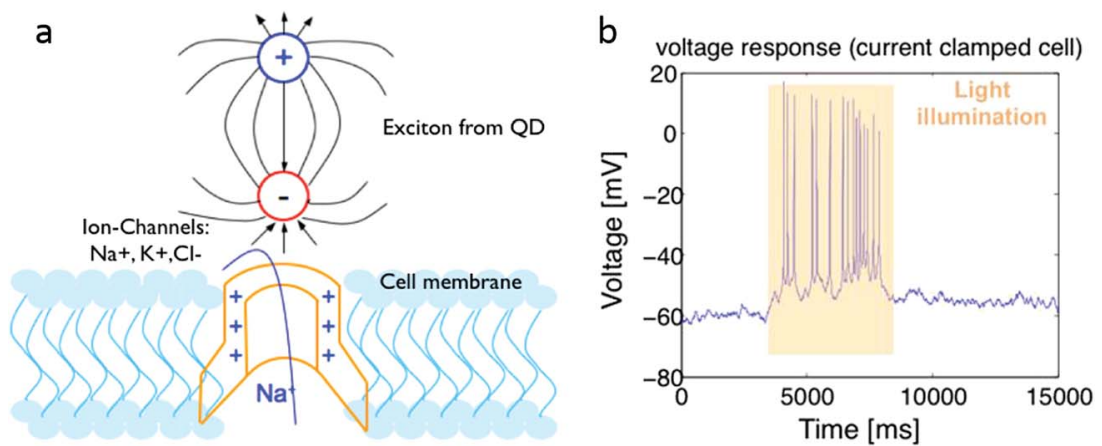

Fig. 7 (a) Stimulating cells through QDs using dipole fields generated under light excitation. (b) Patch-clamp recording of a cortical neuron cultured on a CdSe QDs film stimulated by light. Reprinted with permission from ref. 94.

for optogenetics. Gold nanoparticles, which possessing photothermal property, have also been applied in optogenetics to activate thermal sensitive ion channels. ${ }^{58,95}$ For example, Carvalho-de-Souza et al. triggered neuronal depolarization with gold nanoparticles conjugated to neuronal membrane proteins on exposure to light pulses. ${ }^{96}$ These ligand-conjugated gold nanoparticles enable robust optical triggering of action potentials resistant to washout at low dosage. Magnetic nanoparticles that possess magnetothermal property were driven by alternating magnetic fields and achieved wireless activation of the heat-sensitive capsaicin receptor TRPV1. However, it should be noted that for thermal-sensitive ion channels and "mechanosensor" ion channels (activated by ultrasound or magnetic field $)^{97}$ the spatiotemporal precision is limited due to the relative slow response of these ion channels as well as the slow conversion of the external excitation to the stimuli energy (heat or mechanical deformation). There are also nanomaterials applied for other purposes in optogenetics. Tee et al. used piezoresistive sensors made with carbon nanotube embedded polyurethane elastomer to trigger LED pulse to optogenetically stimulate neurons at high frequency $(200 \mathrm{~Hz}) .^{98}$ Amphiphilic nanofibers formed by aggregation of peptides were used to encapsulate neural progenitor cells, which induced very rapid differentiation of cells into neurons..$^{99}$ Poly(butyl cyanoacrylate) nanomaterials was used for delivery purposes across the bloodbrain barrier (BBB) via intravenous administration. ${ }^{100}$

\section{Challenges and future directions}

Although nanomaterials show great potential in revolutionising optogenetics, with some nanomaterials even having been applied in current research, there are still several challenges pending resolution. Upconversion nanomaterials can be further optimized as the nanotransducer for optogenetics. The emission peaks of upconversion nanomaterials do not fully match with the excitation spectra of currently available light-gated channels, especially for the photoinhibitors. The emission peaks of $\mathrm{NaYF}_{4}: \mathrm{Yb}, \mathrm{Tm}$ at $475 \mathrm{~nm}$ and $\mathrm{NaYF}_{4}: \mathrm{Yb}, \mathrm{Er}$ at $545 \mathrm{~nm}$ match well with popular stimulators $\mathrm{ChR2}$ and cationconducting channelrhodopsin (VChR1) respectively. Unfortunately, for the inhibitor halorhodopsin (NpHR) and archaerhodopsin (Arch), which have maximum excitation at 590 $\mathrm{nm}$ and $566 \mathrm{~nm}$ respectively, conventional upconversion nanomaterials still lack efficient emission peaks at these wavelengths. Due to this constraint, the UCNP-mediated optogenetics is still limited to the stimulation of the neuronal activities, while inhibition has yet to be achieved. Definitely, the development of upconversion nanomaterials that possess emission peaks covering the excitation spectra of optogenetic inhibitor is ardently awaited, which requires the nanoscopic engineering of UCNPs. ${ }^{101,102}$ The new type of TTA-based upconversion nanomaterials may also be applicable for this purpose. ${ }^{103}$ Another possible improvement would be to ensure that the nanomaterial-mediated stimulation/inhibition can occur in a more rapid and precise manner. In the current research, the modulation frequency is limited to $10 \mathrm{~Hz}$, which is much lower than the most rapid gating of $200 \mathrm{~Hz}$ for ChR2. ${ }^{104}$ While the reason why higher frequency modulation results in failed firing of the neuronal activation is not yet known. One possible reason might be that the nanomaterial itself has unique properties of slow response to the excitation and long decay of the luminescence..$^{30,101}$ Therefore, more research needs to be done to reveal the mechanism and enhance manipulation resolution spatiotemporally. The common problem of low luminescence efficiency of the upconversion nanomaterials is also a hindrance in nanomaterials' application in optogenetics. This in turn requires the usage of powerful excitation source (up to several watts), ${ }^{29,30}$ causing the heating problem as well safety concerns. Thus more research efforts in improving the upconverting efficiency of nanomaterials remains crucial.

Other than the upconversion nanomaterials, materials with other properties can also be exploited for optogenetics. With the revelation of thermal-sensitive channels recently, photothermic nanomaterials also show great potential in the field of optogenetics. ${ }^{33,105-107}$ Other than the well-known photothermal gold nanoparticles, nanomaterials that were previously applied in photothermic therapy can also be applied for optogenetics. Among these photothermic materials, $\mathrm{Nd}^{3+}$ doped nanomaterials have been proven to be efficient. ${ }^{108,109}$ Considering that $\mathrm{Nd}^{3+}$ can also serve as the sensitizer for upconversion, it provides a possibility of producing a series of nanomaterials possessing both photothermic and light-upconverting 
properties, thus integrating dual-modality of thermal and optical sensitive stimulations. ${ }^{\mathbf{1 0 8}}$ If extended further, nanomaterials that convert X-ray or ultrasound into luminescence or heat can also be applied if the excitation source is changed from light to X-ray or ultrasound, which also provides revolutionary solutions to the limitation of shallow penetration of light in optogenetics. ${ }^{31}$ Those nanomaterials that serve as the contrast agents for photoacoustic imaging can be conveniently adapted in this application. ${ }^{\mathbf{1 1 0 , 1 1 1}}$

Another issue is the synthesis and modification of the nanomaterials such that they are able to cross the BBB and reach the central nervous system. In recent attempts of nanomaterial-mediated optogenetics, nanomaterials were directly mixed into neuronal culture media or directly injected into the animal model. ${ }^{30,33,105}$ This is acceptable as a conceptual proof in an in vitro demonstration or early stage animal test. However, with the ultimate goal of clinical application, the ability of nanomaterials to cross BBB can never be skipped over. Regardless of their intended applications (ion channel stimulating/inhibiting, neuronal activity sensing, or optogene delivery), nanomaterials must be capable of crossing the BBB, reaching the central nervous system selectively and precisely. Current research has revealed that the two most important factors determining nanomaterials' ability to cross the BBB are the size (usually less than $100 \mathrm{~nm})^{\mathbf{1 1 2}}$ and surface property of the nanoparticles. It is well known that the surface chemistry of a nanoparticle determines its pharmacokinetics, biodistribution and clearance pathway, and PEGylation is required to avoid recognition by the reticuloendothelial system and extend circulation time..$^{65,112}$ Sustained delivery of genetic material with viscous formulations such as supramolecular hydrogels ${ }^{113-117}$ or thermogels ${ }^{118-124}$ could also be useful for prolonged expression of the modified trait by the nerve cells.

However, it should also be noted that some intrinsic disadvantages of nanomaterials hinder their applications in optogenetics. The first issue is the potential toxicity of nanomaterials. Several studies claim that the toxicity of nanomaterials is not obvious by carefully selecting the nanocrystals, elaborate surface modification and using in proper dosage. ${ }^{\mathbf{1 2 5 , 1 2 6}}$ While, there still lacks studies of long-term (e.g. tens of years) toxicity evaluation in mammals to confirm they are safe when applied clinically. Moreover, considering that inorganic crystals are non-biodegradable, their clearance pathways are relatively complicate, either by the renal route (for nanoparticles smaller than $50 \mathrm{~nm}$ ) or by the biliary route (for nanoparticles larger than $50 \mathrm{~nm}) .{ }^{\mathbf{1 2 5}}$ The elimination of these nanomaterials can last for several months, which in turn leads to the concern of nanomaterials accumulated toxicity. The second issue is the targeting efficiency of nanomaterials. Usually nanomaterials is not biological reactive by themselves, thus targeting agents are required to functionalize the nanomaterials. ${ }^{127}$ These targeting agents are usually antibody, peptide or folic acid that specifically binding to or consumed by the targets. Currently, improving the targeting efficiency is a hot topic in the development of biomaterials, which still await for optimizations. The third issue is the non-propagation of nanomaterials-based optogene delivery vector. Virus vectors, insert exotic DNA sequence into the host DNA sequence, thus enabling the propagation while cell proliferation. ${ }^{22}$ However, for nanomaterials vectors, it should be noted that only plasmid DNA or siRNA are usually delivered, and not integrated into the host DNA sequence, thus non-propagative. Although it has been recently reported that cell-penetrating peptides help to insert exotic mitochondrial DNA into host mitochondria for propagation, ${ }^{\mathbf{1 2 8}}$ these researches are still in quite early stage, and far from applicable for the producing of transgenic animals. Addressing of these problems will not only promote the application of nanomaterials in optogenetics, but also benefit their applications in a variety of biological researches.

\section{Conclusion}

With the developments of nanoscience and nanotechnology in the past decades, nanomaterials with new and attractive properties continuously springing up, some of which possess properties that may provide promising solutions to the unmet needs in optogenetics. Incorporating nanomaterials into optogenetics with elaborate design will make optogenetics a more powerful neuroscientific toolkit with deeper light penetration, less invasiveness and more precise spatiotemporal manipulation. This enhancement of optogenetics will boost its applications in neuroscience and beyond, especially in therapeutic and clinical applications.

\section{Acknowledgements}

The authors would like to express gratitude to the $A^{*}$ STAR Personal Care Grant (Project no. 1325400026) for support of this project. K. Huang gratefully acknowledges Dr Qian Lin for the many helpful and constructive discussions.

\section{References}

1 E. S. Boyden, F. Zhang, E. Bamberg, G. Nagel and K. Deisseroth, Nat. Neurosci., 2005, 8, 1263-1268.

2 J. P. Yao, W. S. Hou and Z. Q. Yin, Int. J. Ophthalmol., 2012, 5, 517-522.

3 B. Y. Chow and E. S. Boyden, Sci. Transl. Med., 2013, 5(177), 177 ps5.

4 R. A. McDevitt, S. J. Reed and J. P. Britt, Neuropsychiatr. Dis. Treat., 2014, 10, 1369-1379.

5 K. Yamamoto, Z. Tanei, T. Hashimoto, T. Wakabayashi, H. Okuno, Y. Naka, O. Yizhar, L. E. Fenno, M. Fukayama, H. Bito, J. R. Cirrito, D. M. Holtzman, K. Deisseroth and T. Iwatsubo, Cell Rep., 2015, 11, 859-865.

6 K. Deisseroth, Nat. Methods, 2011, 8, 26-29.

7 J. R. Melchior, M. J. Ferris, G. D. Stuber, D. R. Riddle and S. R. Jones, J. Neurochem., 2015, 134, 833-844.

8 S. Batabyal, G. Cervenka, D. Birch, Y. T. Kim and S. Mohanty, Sci. Rep., 2015, 5, 17857.

9 J. Y. Lin, P. M. Knutsen, A. Muller, D. Kleinfeld and R. Y. Tsien, Nat. Neurosci., 2013, 16, 1499-1508. 
10 B. Y. Chow, X. Han, A. S. Dobry, X. Qian, A. S. Chuong, M. Li, M. A. Henninger, G. M. Belfort, Y. Lin, P. E. Monahan and E. S. Boyden, Nature, 2010, 463, 98-102.

11 J. Wietek, R. Beltramo, M. Scanziani, P. Hegemann, T. G. Oertner and J. Simon Wiegert, Sci. Rep., 2015, 5, 14807.

12 D. Huber, L. Petreanu, N. Ghitani, S. Ranade, T. Hromadka, Z. Mainen and K. Svoboda, Nature, 2008, 451, 61-64.

13 Y. Iwai, S. Honda, H. Ozeki, M. Hashimoto and H. Hirase, Neurosci. Res., 2011, 70, 124-127.

14 S. Royer, B. V. Zemelman, M. Barbic, A. Losonczy, G. Buzsáki and J. C. Magee, Eur. J. Neurosci., 2010, 31, 2279-2291.

15 T.-I. Kim, J. G. McCall, Y. H. Jung, X. Huang, E. R. Siuda, Y. Li, J. Song, Y. M. Song, H. A. Pao, R.-H. Kim, C. Lu, S. D. Lee, I.-S. Song, G. Shin, R. Al-Hasani, S. Kim, M. P. Tan, Y. Huang, F. G. Omenetto, J. A. Rogers and M. R. Bruchas, Science, 2013, 340, 211-216.

16 K. L. Montgomery, A. J. Yeh, J. S. Ho, V. Tsao, S. Mohan Iyer, L. Grosenick, E. A. Ferenczi, Y. Tanabe, K. Deisseroth, S. L. Delp and A. S. Y. Poon, Nat. Methods, 2015, 12, 969974.

17 J. Yoon, M. Lee, K. Lee, N. Kim, J. M. Kim, J. Park, H. Yu, C. Choi, W. D. Heo and Y. Park, Sci. Rep., 2015, 5, 13289.

18 K. Ohki, S. Chung, Y. H. Ch'ng, P. Kara and R. C. Reid, Nature, 2005, 433, 597-603.

19 W. Akemann, H. Mutoh, A. Perron, Y. K. Park, Y. Iwamoto and T. Knopfel, J. Neurophysiol., 2012, 108, 2323-2337.

20 D. A. Storace, O. R. Braubach, L. Jin, L. B. Cohen and U. Sung, Sci. Rep., 2015, 5, 10212.

21 H. Kandori, Front. Mol. Biosci., 2015, 2, 52.

22 C. E. Thomas, A. Ehrhardt and M. A. Kay, Nat. Rev. Genet., 2003, 4, 346-358.

23 N. Nayerossadat, T. Maedeh and P. A. Ali, Adv. Biomed. Res., 2012, 1, 27.

24 J. Akerboom, N. Carreras Calderón, L. Tian, S. Wabnig, M. Prigge, J. Tolö, A. Gordus, M. B. Orger, K. E. Severi, J. J. Macklin, R. Patel, S. R. Pulver, T. J. Wardill, E. Fischer, C. Schüler, T.-W. Chen, K. S. Sarkisyan, J. S. Marvin, C. I. Bargmann, D. S. Kim, S. Kügler, L. Lagnado, P. Hegemann, A. Gottschalk, E. R. Schreiter and L. L. Looger, Front. Mol. Neurosci., 2013, 6, 2.

25 A. P. Alivisatos, A. M. Andrews, E. S. Boyden, M. Chun, G. M. Church, K. Deisseroth, J. P. Donoghue, S. E. Fraser, J. Lippincott-Schwartz, L. L. Looger, S. Masmanidis, P. L. McEuen, A. V. Nurmikko, H. Park, D. S. Peterka, C. Reid, M. L. Roukes, A. Scherer, M. Schnitzer, T. J. Sejnowski, K. L. Shepard, D. Tsao, G. Turrigiano, P. S. Weiss, C. Xu, R. Yuste and X. Zhuang, ACS Nano, 2013, 7, 1850-1866.

26 B. L. Davidson and X. O. Breakefield, Nat. Rev. Neurosci., 2003, 4, 353-364.

27 X. Han and E. S. Boyden, PLoS One, 2007, 2, e299.

28 F. Zhang, M. Prigge, F. Beyriere, S. P. Tsunoda, J. Mattis, O. Yizhar, P. Hegemann and K. Deisseroth, Nat. Neurosci., 2008, 11, 631-633.
29 S. Hososhima, H. Yuasa, T. Ishizuka, M. R. Hoque, T. Yamashita, A. Yamanaka, E. Sugano, H. Tomita and H. Yawo, Sci. Rep., 2015, 5, 16533.

30 S. Shah, J. J. Liu, N. Pasquale, J. Lai, H. McGowan, Z. P. Pang and K. B. Lee, Nanoscale, 2015, 7, 16571-16577.

31 R. Berry, M. Getzin, L. Gjesteby and G. Wang, Photonics, 2015, 2, 23.

32 L. He, Y. Zhang, G. Ma, P. Tan, Z. Li, S. Zang, X. Wu, J. Jing, S. Fang, L. Zhou, Y. Wang, Y. Huang, P. G. Hogan, G. Han and Y. Zhou, eLife, 2015, 4, e10024.

33 J. L. Carvalho-de-Souza, J. S. Treger, B. Dang, S. B. Kent, D. R. Pepperberg and F. Bezanilla, Neuron, 2015, 86, 207217.

34 L. Li, R. Hasan and X. Zhang, J. Neurosci., 2014, 34, 82468258.

35 H. Huang, S. Delikanli, H. Zeng, D. M. Ferkey and A. Pralle, Nat. Nanotechnol., 2010, 5, 602-606.

36 V. Gradinaru, K. R. Thompson, F. Zhang, M. Mogri, K. Kay, M. B. Schneider and K. Deisseroth, J. Neurosci., 2007, 27, 14231-14238.

37 D. Bellmann, A. Richardt, R. Freyberger, N. Nuwal, M. Schwarzel, A. Fiala and K. F. Stortkuhl, Front. Behav. Neurosci., 2010, 4, 27.

38 L. Madisen, T. Mao, H. Koch, J.-m. Zhuo, A. Berenyi, S. Fujisawa, Y.-W. A. Hsu, A. J. Garcia, X. Gu, S. Zanella, J. Kidney, H. Gu, Y. Mao, B. M. Hooks, E. S. Boyden, G. Buzsaki, J. M. Ramirez, A. R. Jones, K. Svoboda, X. Han, E. E. Turner and H. Zeng, Nat. Neurosci., 2012, 15, 793-802.

39 O. Yizhar, L. E. Fenno, T. J. Davidson, M. Mogri and K. Deisseroth, Neuron, 2011, 71, 9-34.

40 J. M. Kralj, A. D. Douglass, D. R. Hochbaum, D. Maclaurin and A. E. Cohen, Nat. Methods, 2012, 9, 90-95.

41 D. Smetters, A. Majewska and R. Yuste, Methods, 1999, 18, 215-221.

42 A. F. Silbering, R. Bell, C. G. Galizia and R. Benton, J. Visualized Exp., 2012, e2976.

43 F. B. Shipley, C. M. Clark, M. J. Alkema and A. M. Leifer, Front. Neural Circuits, 2014, 8, 28.

44 A. M. Packer, L. E. Russell, H. W. P. Dalgleish and M. Hausser, Nat. Methods, 2015, 12, 140-146.

45 E. Ferrea, A. Maccione, L. Medrihan, T. Nieus, D. Ghezzi, P. Baldelli, F. Benfenati and L. Berdondini, Front. Neural Circuits, 2012, 6, 80.

46 C. E. Rowland, K. Susumu, M. H. Stewart, E. Oh, A. J. Mäkinen, T. J. O'Shaughnessy, G. Kushto, M. A. Wolak, J. S. Erickson, A. L. Efros, A. L. Huston and J. B. Delehanty, Nano Lett., 2015, 15, 6848-6854.

47 J. D. Marshall and M. J. Schnitzer, ACS Nano, 2013, 7, 46014609.

48 A. J. Hallock, P. L. Redmond and L. E. Brus, Proc. Natl. Acad. Sci. U. S. A., 2005, 102, 1280-1284.

49 J. Zhang, T. Atay and A. V. Nurmikko, Nano Lett., 2009, 9, 519-524.

50 C. Lumdee, S. Toroghi and P. G. Kik, ACS Nano, 2012, 6, 6301-6307. 
51 Q. Yan, J. Yuan, Z. Cai, Y. Xin, Y. Kang and Y. Yin, J. Am. Chem. Soc., 2010, 132, 9268-9270.

52 R. Liang, T. Yin and W. Qin, Anal. Chim. Acta, 2015, 853, 291-296.

53 V. Pachauri, K. Kern and K. Balasubramanian, Appl. Phys. Lett., 2013, 102, 023501.

54 A. B. Kharitonov, A. N. Shipway and I. Willner, Anal. Chem., 1999, 71, 5441-5443.

55 X. J. Loh and Y. L. Wu, Chem. Commun., 2015, 51, 1081510818.

56 S. Jiang, Y. Z. Poh and X. J. Loh, RSC Adv., 2015, 5, 7132271328.

57 C. K. Liu, Q. Dou, S. S. Liow, J. N. Kumar and X. J. Loh, Aust. J. Chem., 2016, 69, 363-371.

58 X. J. Loh, T. C. Lee, Q. Dou and G. R. Deen, Biomater. Sci., 2015, 4, 70-86.

59 X. J. Loh, A. A. Karim and C. Owh, J. Mater. Chem. B, 2015, 3, 7641-7652.

60 S. Jiang, D. Kai, Q. Q. Dou and X. J. Loh, J. Mater. Chem. B, 2015, 3, 6897-6904.

61 X. J. Loh, S. J. Ong, Y. T. Tung and H. T. Choo, Macromol. Biosci., 2013, 13, 1092-1099.

62 X. J. Loh, S. J. Ong, Y. T. Tung and H. T. Choo, Mater. Sci. Eng., C, 2013, 33, 4545-4550.

63 X. J. Loh, J. Appl. Polym. Sci., 2013, 127, 992-1000.

$64 \mathrm{~J} . \mathrm{Li}$ and X. J. Loh, Adv. Drug Delivery Rev., 2008, 60, 10001017.

65 A. Mangraviti, S. Y. Tzeng, K. L. Kozielski, Y. Wang, Y. Jin, D. Gullotti, M. Pedone, N. Buaron, A. Liu, D. R. Wilson, S. K. Hansen, F. J. Rodriguez, G.-D. Gao, F. DiMeco, H. Brem, A. Olivi, B. Tyler and J. J. Green, ACS Nano, 2015, 9, 1236-1249.

66 M. K. G. Jayakumar, A. Bansal, K. Huang, R. Yao, B. N. Li and Y. Zhang, ACS Nano, 2014, 8, 4848-4858.

67 F. Zhang, J. Vierock, O. Yizhar, L. E. Fenno, S. Tsunoda, A. Kianianmomeni, M. Prigge, A. Berndt, J. Cushman and J. Polle, Cell, 2011, 147, 1446-1457.

68 A. Punjabi, X. Wu, A. Tokatli-Apollon, M. El-Rifai, H. Lee, Y. Zhang, C. Wang, Z. Liu, E. M. Chan, C. Duan and G. Han, ACS Nano, 2014, 8, 10621-10630.

69 G. Chen, J. Shen, T. Y. Ohulchanskyy, N. J. Patel, A. Kutikov, Z. Li, J. Song, R. K. Pandey, H. Ågren, P. N. Prasad and G. Han, ACS Nano, 2012, 6, 8280-8287.

70 Z. Li, Y. Zhang, X. Wu, L. Huang, D. Li, W. Fan and G. Han, J. Am. Chem. Soc., 2015, 137, 5304-5307.

71 Z. Li, Y. Zhang, X. Wu, X. Wu, R. Maudgal, H. Zhang and G. Han, Adv. Sci., 2015, 2, 1500001.

72 A. Bansal, H. Liu, M. K. Jayakumar, S. Andersson-Engels and Y. Zhang, Small, 2016, 12, 1732-1743.

73 Y. Zhang, L. Huang, Z. Li, G. Ma, Y. Zhou and G. Han, ACS Nano, 2016, 10, 3881-3885.

$74 \mathrm{X} . \mathrm{Wu}, \mathrm{G}$. Chen, J. Shen, Z. Li, Y. Zhang and G. Han, Bioconjugate Chem., 2015, 26, 166-175.

75 L. Liang, H. Wu, H. Hu, M. Wu and Q. Su, J. Alloys Compd., 2004, 368, 94-100.

76 K. W. Krämer, D. Biner, G. Frei, H. U. Güdel, M. P. Hehlen and S. R. Lüthi, Chem. Mater., 2004, 16, 1244-1251.
77 X. F. Wang, X. H. Yan, C. X. Kan, K. L. Ma, Y. Xiao and S. G. Xiao, Appl. Phys. B: Lasers Opt., 2010, 101, 623-629.

78 J. Shen, G. Chen, T. Y. Ohulchanskyy, S. J. Kesseli, S. Buchholz, Z. Li, P. N. Prasad and G. Han, Small, 2013, 9, 3213-3217.

79 J. Shen, G. Chen, A.-M. Vu, W. Fan, O. S. Bilsel, C.-C. Chang and G. Han, Adv. Opt. Mater., 2013, 1, 644-650.

80 Y.-F. Wang, G.-Y. Liu, L.-D. Sun, J.-W. Xiao, J.-C. Zhou and C.-H. Yan, ACS Nano, 2013, 7, 7200-7206.

81 X. Xie, N. Gao, R. Deng, Q. Sun, Q.-H. Xu and X. Liu, J. Am. Chem. Soc., 2013, 135, 12608-12611.

82 H. Wen, H. Zhu, X. Chen, T. F. Hung, B. Wang, G. Zhu, S. F. Yu and F. Wang, Angew. Chem., Int. Ed., 2013, 52, 13419-13423.

83 Y. Zhong, G. Tian, Z. Gu, Y. Yang, L. Gu, Y. Zhao, Y. Ma and J. Yao, Adv. Mater., 2014, 26, 2831-2837.

84 X. Wu, H. Lee, O. Bilsel, Y. Zhang, Z. Li, T. Chen, Y. Liu, C. Duan, J. Shen, A. Punjabi and G. Han, Nanoscale, 2015, 7, 18424-18428.

85 G. Chen, J. Damasco, H. Qiu, W. Shao, T. Y. Ohulchanskyy, R. R. Valiev, X. Wu, G. Han, Y. Wang, C. Yang, H. Ågren and P. N. Prasad, Nano Lett., 2015, 15, 7400-7407.

86 P. Anikeeva and K. Deisseroth, Google Patents, p. PCT/ US2011/059287, Nov 42011.

87 http://www.eurekalert.org/pub_releases/2013-10/uommegt102413.php.

88 S. Shah, J.-J. Liu, N. Pasquale, J. Lai, H. McGowan, Z. P. Pang and K.-B. Lee, Nanoscale, 2015, 7, 16571-16577.

89 X. Wu, Y. Zhang, K. Takle, O. Bilsel, Z. Li, H. Lee, Z. Zhang, D. Li, W. Fan, C. Duan, E. M. Chan, C. Lois, Y. Xiang and G. Han, ACS Nano, 2016, 10, 1060-1066.

90 S. Hososhima, H. Yuasa, T. Ishizuka and H. Yawo, Proc. SPIE, 2015, 9305.

91 Q. Dou and Y. Zhang, Langmuir, 2011, 27, 13236-13241.

92 Q. Dou, N. M. Idris and Y. Zhang, Biomaterials, 2013, 34, 1722-1731.

93 M. Dahan, S. Levi, C. Luccardini, P. Rostaing, B. Riveau and A. Triller, Science, 2003, 302, 442-445.

94 K. Lugo, X. Miao, F. Rieke and L. Y. Lin, Biomed. Opt. Express, 2012, 3, 447-454.

95 E. Y. Ye, M. D. Regulacio, S. Y. Zhang, X. J. Loh and M. Y. Han, Chem. Soc. Rev., 2015, 44, 6001-6017.

96 J. L. Carvalho-de-Souza, J. S. Treger, B. Dang, S. B. Kent, D. R. Pepperberg and F. Bezanilla, Neuron, 2015, 86, 207217.

97 S. Ibsen, A. Tong, C. Schutt, S. Esener and S. H. Chalasani, Nat. Commun., 2015, 6, 8264.

98 B. C.-K. Tee, A. Chortos, A. Berndt, A. K. Nguyen, A. Tom, A. McGuire, Z. C. Lin, K. Tien, W.-G. Bae, H. Wang, P. Mei, H.-H. Chou, B. Cui, K. Deisseroth, T. N. Ng and Z. Bao, Science, 2015, 350, 313-316.

99 G. A. Silva, C. Czeisler, K. L. Niece, E. Beniash, D. A. Harrington, J. A. Kessler and S. I. Stupp, Science, 2004, 303, 1352-1355.

100 S. E. Gelperina, A. S. Khalansky, I. N. Skidan, Z. S. Smirnova, A. I. Bobruskin, S. E. Severin, B. Turowski, 
F. E. Zanella and J. Kreuter, Toxicol. Lett., 2002, 126, 131141.

101 K. Huang, N. M. Idris and Y. Zhang, Small, 2015, 836-852. 102 F. Wang and X. Liu, Acc. Chem. Res., 2014, 47, 1378-1385.

103 T. N. Singh-Rachford and F. N. Castellano, Coord. Chem. Rev., 2010, 254, 2560-2573.

104 L. A. Gunaydin, O. Yizhar, A. Berndt, V. S. Sohal, K. Deisseroth and P. Hegemann, Nat. Neurosci., 2010, 13, 387-392.

105 R. Chen, G. Romero, M. G. Christiansen, A. Mohr and P. Anikeeva, Science, 2015, 347, 1477-1480.

106 B. Nilius and G. Owsianik, Genome Biol., 2011, 12, 218.

107 A. D. Güler, H. Lee, T. Iida, I. Shimizu, M. Tominaga and M. Caterina, J. Neurosci., 2002, 22, 6408-6414.

108 E. Carrasco, B. del Rosal, F. Sanz-Rodríguez, Á. J. de la Fuente, P. H. Gonzalez, U. Rocha, K. U. Kumar, C. Jacinto, J. G. Solé and D. Jaque, Adv. Funct. Mater., 2015, 25, 615-626.

109 U. Rocha, K. Upendra Kumar, C. Jacinto, J. Ramiro, A. J. Caamaño, J. García Solé and D. Jaque, Appl. Phys. Lett., 2014, 104, 053703.

110 A. De La Zerda, C. Zavaleta, S. Keren, S. Vaithilingam, S. Bodapati, Z. Liu, J. Levi, B. R. Smith, T.-J. Ma, O. Oralkan, Z. Cheng, X. Chen, H. Dai, B. T. Khuri-Yakub and S. S. Gambhir, Nat. Nanotechnol., 2008, 3, 557-562.

111 A. A. Oraevsky, J. Photoacoust., 2015, 3, 1-2.

112 J. W. Seo, J. Ang, L. M. Mahakian, S. Tam, B. Fite, E. S. Ingham, J. Beyer, J. Forsayeth, K. S. Bankiewicz, T. Xu and K. W. Ferrara, J. Controlled Release, 2015, 220, 51-60.

113 H. Ye, C. Owh, S. Jiang, C. Ng, D. Wirawan and X. Loh, Polymers, 2016, 8, 130.
114 A. Abdul Karim, Q. Dou, Z. Li and X. J. Loh, Chem.-Asian J., 2016, 11(9), 1300-1321.

115 H. Ye, C. Owh and X. J. Loh, RSC Adv., 2015, 5, 48720-48728.

116 A. A. Karim and X. J. Loh, Soft Matter, 2015, 11, 5425-5434.

117 D. Kai, Z. W. Low, S. S. Liow, A. A. Karim, H. Y. Ye, G. R. Jin, K. Li and X. J. Loh, ACS Sustainable Chem. Eng., 2015, 3, 2160-2169.

118 Y.-L. Wu, H. Wang, Y.-K. Qiu and X. J. Loh, RSC Adv., 2016, 6, 44506-44513.

119 S. S. Liow, Q. Dou, D. Kai, A. A. Karim, K. Zhang, F. Xu and X. J. Loh, ACS Biomater. Sci. Eng., 2016, 2, 295-316.

120 Z. B. Li and X. J. Loh, Chem. Soc. Rev., 2015, 44, 2865-2879.

121 X. J. Loh, H. X. Gan, H. Wang, S. J. E. Tan, K. Y. Neoh, S. S. J. Tan, H. F. Diong, J. J. Kim, W. L. S. Lee, X. T. Fang, O. Cally, S. S. Yap, K. P. Liong and K. H. Chan, J. Appl. Polym. Sci., 2014, 131, 39924.

122 X. J. Loh, W. Guerin and S. M. Guillaume, J. Mater. Chem., 2012, 22, 21249-21256.

123 V. P. N. Nguyen, N. Y. Kuo and X. J. Loh, Soft Matter, 2011, 7, 2150-2159.

124 X. J. Loh, P. N. N. Vu, N. Y. Kuo and J. Li, J. Mater. Chem., 2011, 21, 2246-2254.

125 Y. Sun, W. Feng, P. Yang, C. Huang and F. Li, Chem. Soc. Rev., 2015, 44, 1509-1525.

126 F. M. Winnik and D. Maysinger, Acc. Chem. Res., 2013, 46, 672-680.

127 J. G. Huang, T. Leshuk and F. X. Gu, Nano Today, 2011, 6, 478-492.

128 Y. G. Yoon, M. D. Koob and Y. H. Yoo, Adv. Anat., 2010, 43, 97-109. 